\title{
THE CONNECTIVITY OF MULTICURVES DETERMINED BY INTEGRAL WEIGHT TRAIN TRACKS
}

\author{
ANDREW HAAS AND PERRY SUSSKIND
}

\begin{abstract}
An integral weighted train track on a surface determines the isotopy class of an embedded closed 1-manifold. We are interested in the connectivity of the resulting 1-manifold. In general there is an algorithm for determining connectivity, and in the simplest case of a 2-parameter train track on a surface of genus one there is an explicit formula. We derive a formula for the connectivity of the closed 1-manifold determined by a 4-parameter train track on a surface of genus two which is computable in polynomial time. We also give necessary and sufficient conditions on the parameters for the resulting 1-manifold to be connected.
\end{abstract}

The graph $\tau$ on the surface $N$ of genus two shown in Figure 1.1 is an example of a train track. When nonnegative integer weights are associated to $\tau$ so that "switching conditions" at the vertices are respected then the resulting weighted train track will determine the nonoriented isotopy class of a closed one-manifold embedded in $N$. Our aim is to describe the connectivity of this one-manifold for given weights.

Let $S(N)$ denote the set of isotopy classes of closed one-manifolds embedded in $N$. The elements of $S(N)$ are called multiple curves. Suppose that a nonnegative integer has been associated to each edge of $\tau$. These weights determine a multiple curve by running the designated number of arcs parallel to each ede of $\tau$ and gluing them together at the vertices.

Switching conditions [2] are needed to guarantee that the arcs will glue together properly at a vertex. For any train track $\tau$, the edges meeting a particular vertex $v$ are all tangent. Choose an orientation for the tangency at $v$. Let $k_{i}$ for $i=i, \ldots, n$ be the weights for those edges which are oriented positively as they approach $v$ and let $l_{j}$ for $j=1, \ldots, m$ be the weights of the edges which are oriented negatively as they approach $v$. The switching condition at the vertex $v$ is $l_{1}+\cdots+l_{m}=k_{1}+\cdots+k_{n}$. For the trivalent vertex of Figure $1.2 \mathrm{a}$ the switching condition takes the form $a+b=c$. The situation where $(a, b, c)=(2,3,5)$ is shown in $1.2 \mathrm{~b}$.

An assignment of nonnegative integral weights to $\tau$ that satisfies the switching condition at each vertex is called a weighting on $\tau$. In particular the as-

Received by the editors June 10, 1989 and, in revised form, November 17, 1989.

1980 Mathematics Subject Classification (1985 Revision). Primary 57N99, 30 F99.

Key words and phrases. Train track, surface topology, multiple curves.

Research supported in part by NSF Grant No. DMS-8702868. 


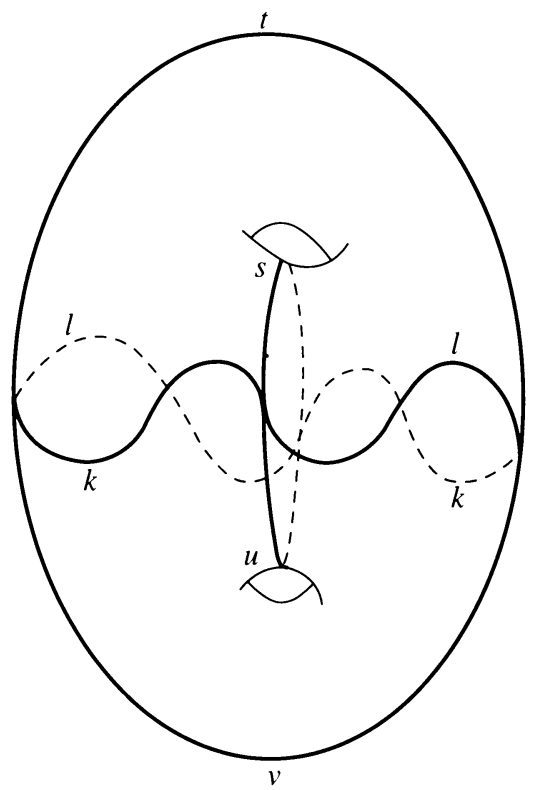

Figure 1.1. The train track $\tau$ on a surface of genus two

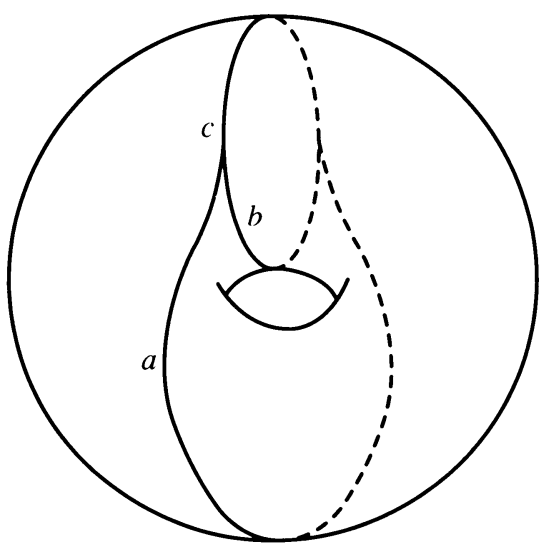

Figure 1.2a

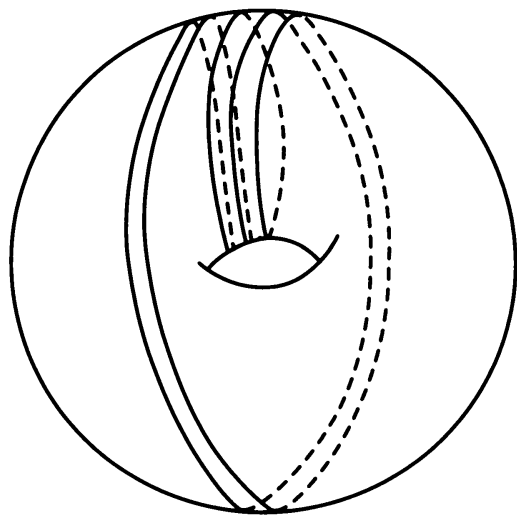

FIGURE 1.2b

signment of positive integer weights $u, v, s, t, k, l$ to the branches of $\tau$ with $l+u=s+k$ and $l+t=v+k$ satisfies the switching conditions and therefore uniquely determines a weighting on $\tau$. We choose to work with the parameters $t, v, k$ and $n=s+t=u+v$ which parameterize all weightings on $\tau$.

Let $M=M(n, t, v, k)$ denote the multiple curve determined by the weighting on $\tau$ with given values $n, t, v, k$ and the remaining values determined from the switching conditions. Let $G=\operatorname{gcd}(v-t+2 k, n)$ and let $\|x\|$ denote the greatest integer less than or equal to $x$. Our main result is the following.

1.1 Theorem. The number of connected components of $M(n, t, v, k)$ is given 
by

where

$$
G-\left|\Sigma\left(\left\|\frac{v-1}{2}\right\|\right)-\Sigma\left(\left\|\frac{v-1}{2}\right\|+k\right)\right|
$$

$$
\Sigma(x)= \begin{cases}x_{0} & \text { if } 0 \leq x_{0}<\left\|\frac{G+1}{2}\right\|, \\ G-(v \bmod 2)-x_{0} & \text { if }\left\|\frac{G+1}{2}\right\| \leq x_{0}<G\end{cases}
$$

and $x_{0}=x \bmod G$.

As a consequence of Theorem 1 we can determine for which $n, t, v$ and $k$ $M(n, t, v, k)$ is connected.

1.2 Theorem. $M(n, t, v, k)$ is connected if and only if either

(i) $\operatorname{gcd}(v-t+2 k, n)=1$ or,

(ii) $\operatorname{gcd}(v-t+2 k, n)=2, t$ and $v$ are even and $k$ is odd.

Furthermore, in case (i) $M$ is a nondividing curve and in case (ii) $M$ divides the surface.

Consider the multiple curves $M(k)=M(n, t, v, k)$ where $n, t$ and $v$ are fixed.

1.3 Theorem. The function $M(k)$ is periodic with period $2 n$ and for any nonnegative integer $w \geq t-v$ the number of integers $k$ with $w \leq k \leq w+2 n-1$ for which $M(k)$ is connected is

$$
\begin{aligned}
& 2 \phi(n) \text { for } n \text { odd, } \\
& n \text { even, } n / 2 \text { odd, } t \text { and } v \text { even, } \\
& n \text { even, } n / 2 \text { even, } t, v \text { and } \frac{v-t}{2} \text { even, } \\
& 4 \phi(n) \text { for } \quad \begin{array}{l}
n \text { even, } v-t \text { odd }, \\
\text { otherwise, }
\end{array}
\end{aligned}
$$

where $\phi(n)$ is the number of integers in $\{0, \ldots, n-1\}$ relatively prime to $n$.

Train tracks originated with William Thurston in his work on diffeomorphisms of surfaces and hyperbolic 3-manifolds [4].

1.1. In order to clarify the definition of $\tau$ and to highlight an important additional property of the train track we will give two alternative descriptions of $\tau$, both of which are of a planar character.

Let $D$ be the closure of the region in the plane interior to the circle of radius ten centered at the origin and exterior to the two circles of radii 1 centered at the points $5 i$ and $-5 i$. Let $D^{\prime}$ be a second copy of $D$. We glue together $D$ and $D^{\prime}$ by identifying the point $(z, D)$ on the boundary of $D$ to the point $\left(-\bar{z}, D^{\prime}\right)$ on the boundary of $D^{\prime}$. Let $J$ be the Möbius transformation of order two with fixed points $10 i$ and $-10 i$. The region $J\left(D^{\prime}\right) \cup D$ is produced by gluing $D$ and $D^{\prime}$ along their outer boundaries, and gives local coordinates in a neighborhood of an outer boundary point. One similarly defines local coordinates in the neighborhood of an inner boundary point. The result of the identification is a compact Riemann surface $N$ of genus two.

The train track $\tau$ when restricted to the two planar subsurfaces $D$ and $D^{\prime}$ of $N$ may be very explicitly described as the union of the graph of the equation $y=-\sin (2 \pi x / 10)$, the segment of the $y$ axis between $4 i$ and $-4 i$, and the outer boundary circle. 
Every Riemann surface of genus two is known to admit a unique hyperelliptic involution $\phi$. On the surface $N$ under consideration $\phi$ is the map interchanging $(z, D)$ and $\left(z, D^{\prime}\right)$. It is quite clear from the construction that $\phi(\tau)=\tau$. The conformal structure also determines a hyperbolic metric for $N$ in which $\phi$ is an isometry.

The quotient of $N$ by the involution $\phi$ is a surface $S$ which is conformally a sphere with six order two branch points. Since $\tau$ is $\phi$-invariant it projects to a graph $\tau_{0}$ on $S$ that has terminal vertices at the branch points of $S . \tau_{0}$ is a train track with stops [3]. In Figures 1.3a, b, c, d we illustrate the sequence of deformations of $\tau_{0}$ that result in the form we will be working with. A weighting for $\tau_{0}$ is, as before, an assignment of nonnegative integers to the edges of $\tau_{0}$ so that the switching conditions are satisfied at the nonterminal vertices.

Let $B$ denote the set of branch points on $S$. A multiarc is defined as the isotopy class relative to $B$ of a closed one manifold, possibly with boundary, embedded in $S$ so that the boundary points belong to $B$. A weighting on $\tau_{0}$

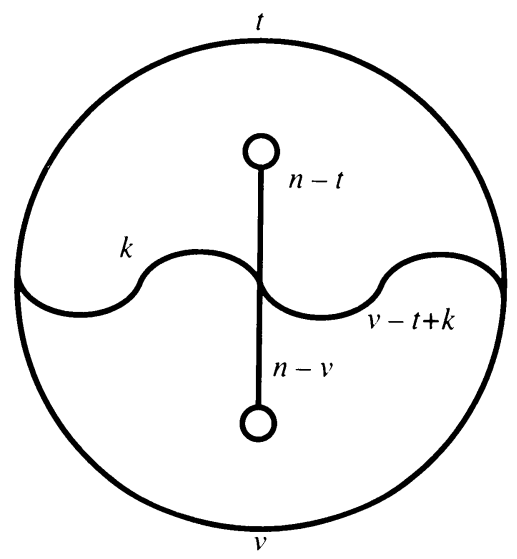

FIGURE 1.3a. The surface $D$

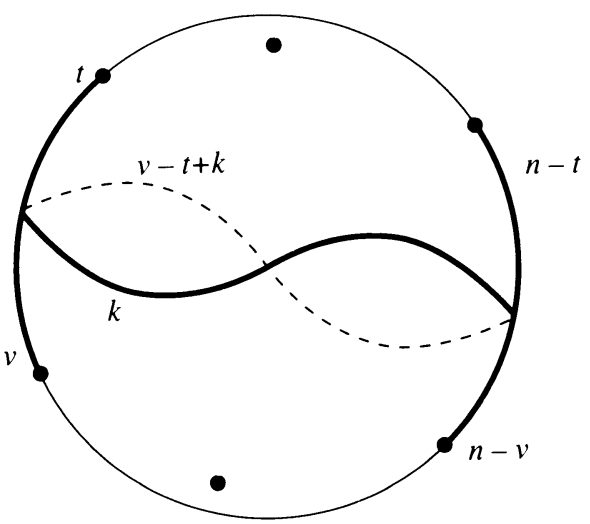

Figure $1.3 \mathrm{~b}$. The quotient surface $S$ with the projected train track $\tau_{0}$ 


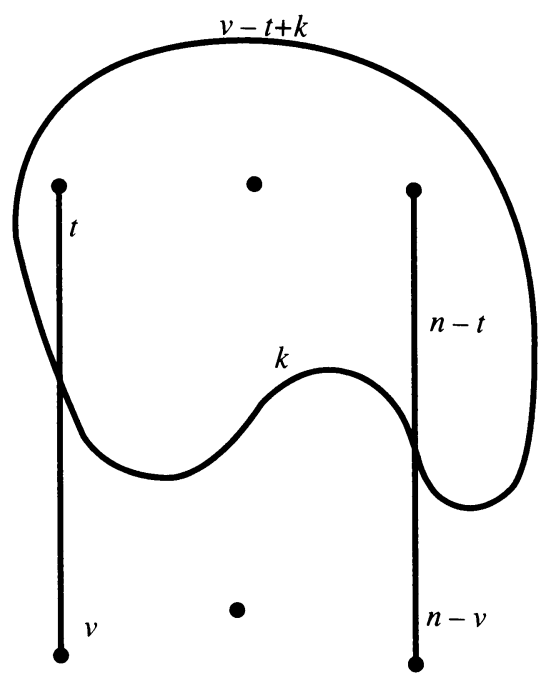

Figure 1.3c. $\tau_{0}$ in the plane

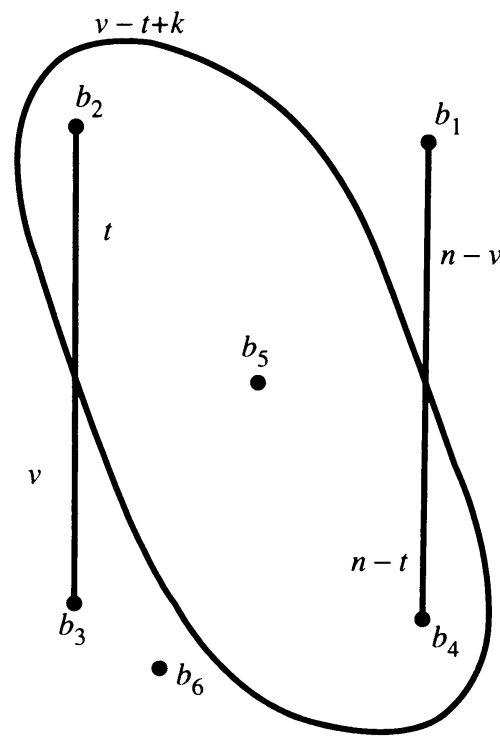

FIGURE 1.3d. $\tau_{0}$ after a counterclockwise half turn of the right vertical segment

will determine a multiarc in the following manner. Choose a regular neighborhood $U$ of $\tau_{0}$. Run a number of arcs corresponding to the given weight parallel to each edge of $\tau$ in $U$. At a nonterminal vertex the arcs will glue together as before. In a neighborhood of a terminal vertex $b$ the procedure may be modeled in the plane by letting $b$ lie at the origin and by making the negative imaginary axis represent the edge $e$ of $\tau_{0}$ terminating at $b$. Let $n$ be the weight for $e$ and suppose that all other branch points are safely out of the picture. The arcs parallel to $e$ are the vertical rays in the lower half plane with endpoints $-1+2 k /(n-1)$ where $0 \leq k \leq n-1$. The endpoints are then 
joined by the semicircles in the upper half plane centered at the origin with radii $1-2 k /(n-1)$ for $0 \leq k<(n-1) / 2$.

A selection of nonnegative integers $n, t, v, k$ with $v-t+k \geq 0, n \geq t$ and $n \geq v$ (Figure 1.3d) determines a weighting on $\tau_{0}$. This weighting may be lifted to a weighting for $\tau$ by giving an edge $e$ of $\tau$ the same weight that $\pi(e)$ has where $\pi: N \rightarrow S$ is the quotient map. Similarly a weighting on $\tau$ projects to a weighting on $\tau_{0}$. The multiarc determined by a weighting on $\tau_{0}$ will lift to the multicurve determined by the lift of the weighting to $\tau$. It follows that the multiarc determined by a weighting on $\tau$ is $\phi$-invariant.

We shall now outline the proof of Theorem 1 . Given a weighting on $\tau$ we transfer this to a weighting on $\tau_{0}$. Let $M_{0}$ be the multiarc determined by the weighting on $\tau_{0}$. The first step in the argument is to determine the connectivity of $M_{0}$. A technique is developed in $\S 2$ that allows us to compute the connectivity for a special class of multiarcs in the plane. In $\S 3$ we see that a multiarc carried by $\tau_{0}$ belongs to this class and so the first step is complete. It then remains to determine the number of components in the lift of $M_{0}$ to $N$. Since $\pi: N \rightarrow S$ is a 2-sheeted branched covering, a component of $M_{0}$ will have either one or two preimages on $N$. This number can be determined from the position of the components on $S$ relative to the branch points. The same data also indicates whether or not the preimage will be a dividing curve on $N$. At the end of $\S 3$ the loose ends are tied together completing the proof of Theorem 1.1. The proofs of Theorem 1.2 and 1.3 appear in $\S 4$.

The train track $\tau$ that we have chosen to work with has the nice property of being invariant under the hyperelliptic involution $\phi$. In [1] we show that every simple closed geodesic on a Riemann surface of genus two is mapped onto itself by the hyperelliptic involution. Thus $\phi$-invariance is less a quirk of the train track than of the genus two surface on which the train track lies.

2

In this section we shall consider a connectivity problem for a family of curves in the plane that are constructed as finite unions of circular arcs and rays. This problem will be resolved for certain special cases that correspond to the multi$\operatorname{arcs} M_{0}(n, t, v, k)$.

Let $U$ and $L$ denote respectively the upper and lower half-planes. The general problem is to determine the connectivity of a set $\mathfrak{L}$ in $\mathbf{C}$ with the following properties:

(1) The closure of a component of $\mathfrak{L} \backslash \mathbf{R}$ is either a semicircle orthogonal to $\mathbf{R}$, or a line segment of length $1 / 2$ orthogonal to $\mathbf{R}$.

(2) There is an integer $N>0$ so that

$$
(\overline{\mathfrak{L} \cap U}) \cap \mathbf{R}=(\overline{\mathfrak{L} \cap L}) \cap \mathbf{R}=\{0,1, \ldots, N-1\} .
$$

$\mathfrak{L}$ is called a labyrinth.

We will further limit the structure of the set $\mathfrak{L}$ by stipulating that

(3) if $C$ is a semicircle in $\mathfrak{L} \cap U(\mathfrak{L} \cap L)$ having endpoints $a$ and $b$ with $a<b$, then for each integer $i$ with $0<i<(b-a) / 2$ the semicircle $C_{i}$ in $\mathfrak{L} \cap U(\mathfrak{L} \cap K)$ with endpoints $a+i$ and $b-i$ also belongs to $\mathfrak{L}$. We refer to such a set $\mathfrak{L}$ as a concentric labyrinth.

Define an equivalence relation on the components of $\mathfrak{L} \cap U(\mathfrak{L} \cap L)$ where two semicircles of $\mathfrak{L} \cap U(\mathfrak{L} \cap L)$ are equivalent if they lie on concentric circles, 
and a line segment $l$ is equivalent to a semicircle $C$ if $l$ is contained in the bounded region in the complement of $C$ in $U(L)$. Note that condition (3) in the definition of concentric labyrinth guarantees that there can be at most one line segment in any equivalence class. We will refer to the elements of any one equivalence class in $U$ or $L$ as a mound.

The equivalence relations defined on $\mathfrak{L} \cap U$ and $\mathfrak{L} \cap L$ give rise to two equivalence relations and hence two partitions of $\{0, \ldots, N-1\}$, the set of endpoints of the curves of $\mathfrak{L} \cap U$ and $\mathfrak{L} \cap L$ in $\mathbf{R}$. From property (3) in the definition we observe that any set of equivalent integers in one of the above partitions is a union of consecutive integers. Thus the equivalence classes of $U \cap \mathfrak{L}$ and $L \cap \mathfrak{L}$ are naturally ordered by the magnitudes of the endpoints. Therefore the labyrinth may be specified by two vectors of positive integers $\mathbf{a}=\left(a_{1}, \ldots, a_{n}\right)$ and $\mathbf{b}=\left(b_{1}, \ldots, b_{n}\right)$ with $\sum a_{i}=\sum b_{i}=N$, where the value $a_{i}\left(b_{i}\right)$ is the number of endpoints in the equivalence class of $\mathfrak{L}$ in $U(L)$ which is $i$ th in the ordering of equivalence classes. We write this labyrinth $\mathfrak{L}(\mathbf{a} ; \mathbf{b})$. The labyrinth $\mathfrak{L}(2,5,4 ; 4,7)$ is shown in Figure 2.1 .

2.1. We now state and prove several lemmas which enable us to determine the connectivity of a concentric labyrinth $\mathfrak{L}$. Lemma 2.1 treats those concentric labyrinths which consist of two mounds in one half plane and one mound in the other. Lemma 2.4 treats the case of three mounds in one of the half planes where the middle mound is a line segment and one mound in the other half plane. Lemmas 2.1, 2.4 and their corollaries will allow us to find the connectivity of a labyrinth which arises from an integral weighting on the train track $\tau_{0}$ defined in $\S 1$.

2.1 Lemma. There is an isotopy of the plane that maps the labyrinth $\mathfrak{L}\left(a_{1}, a_{2} ; b\right)$ onto the labyrinth $\mathfrak{L}(c ; c)$ where $c=\operatorname{gcd}\left(a_{1}, a_{2}\right)$.

The lemma has two immediate consequences. Let $K(\mathfrak{L})$ denote the number of connected components of $\mathfrak{L}$, i.e. the connectivity of $\mathfrak{L}$. Let $A(\mathfrak{L})$ be the number of components of $\mathfrak{L}$ that are arcs, i.e., not closed curves.

2.2 Corollary. $K\left[\mathfrak{L}\left(a_{1}, a_{2} ; b\right)\right]=\left\|\frac{1}{2}(c+1)\right\|$, where $c=\operatorname{gcd}\left(a_{1}, a_{2}\right)$.

2.3 Corollary. $\operatorname{gcd}\left(a_{1}, a_{2}\right)=2 K(\mathfrak{L})-A(\mathfrak{L})$, where $\mathfrak{L}=\mathfrak{L}\left(a_{1}, a_{2} ; b\right)$.

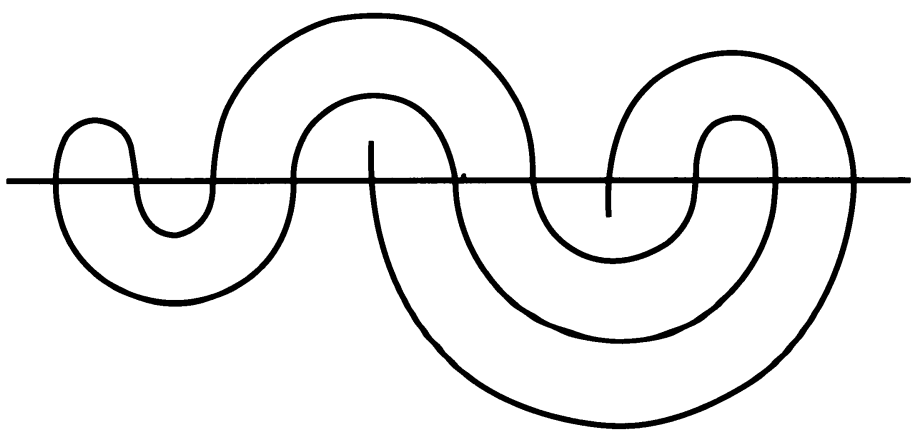

FIGURE 2.1. $\mathfrak{L}(2,5,4 ; 4,7)$ 
Proof of Lemma 2.1. Given $\mathbf{a}=\left(a_{1}, a_{2}\right) \in \mathbf{N}^{2}$ define

$$
\phi(\mathbf{a})= \begin{cases}\left(a_{2}, a_{1}-a_{2}\right), & a_{1}>a_{2}, \\ \left(a_{2}-a_{1}, a_{1}\right), & a_{1}<a_{2}, \\ a_{1}, & a_{1}=a_{2} .\end{cases}
$$

Let $\phi^{n}$ denote the $n$-fold composition of $\phi$ with itself and for any vector $\mathbf{v}=$ $\left(v_{1}, \ldots, v_{n}\right)$ and let $|\mathbf{v}|=\sum_{i=1}^{n} v_{i}$. Since for $\mathbf{a}=\left(a_{1}, a_{2}\right) \in \mathbf{N}^{2},|\phi(\mathbf{a})|<|\mathbf{a}|$, there is an integer $k$ for which the entries of $\phi^{k}(\mathbf{a})$ are equal. Let $c=\phi^{k+1}(\mathbf{a}) \in$ N. It is easily seen that $m=\operatorname{gcd}\left(a_{1}, a_{2}\right)$ if and only if $m=\operatorname{gcd}\left(\phi\left(a_{1}, a_{2}\right)\right)$ (or $m=\phi\left(a_{1}, a_{2}\right)$ if $\left.a_{1}=a_{2}\right)$. It follows that $c=\operatorname{gcd}\left(a_{1}, a_{2}\right)$.

For a labyrinth $\mathfrak{L}=\mathfrak{L}\left(a_{1}, a_{2} ; b\right)$ define $\theta(\mathfrak{L})=\mathfrak{L}\left(\max \left\{a_{1}, a_{2}\right\} ; \phi(\mathbf{a})\right)$, where $\mathbf{a}=\left(a_{1}, a_{2}\right)$. Similarly when $\mathfrak{L}=\mathfrak{L}\left(b ; a_{1}, a_{2}\right)$ define

$$
\theta(\mathfrak{L})=\mathfrak{L}\left(\phi(\mathbf{a}) ; \max \left\{a_{1}, a_{2}\right\}\right) .
$$

$\theta(\mathfrak{L})$ is called an unravelling of $\mathfrak{L}$.

Beginning with $\mathfrak{L}_{0}=\mathfrak{L}\left(a_{1}, a_{2} ; b\right)$ we define a finite sequence $\left\{\mathfrak{L}_{i}\right\}$ of unravellings by $\mathfrak{L}_{i+1}=\theta\left(\mathfrak{L}_{i}\right)$. The sequence will terminate when we reach a labyrinth $\mathfrak{L}_{n}=\mathfrak{L}(c ; c)$ with $c=\operatorname{gcd}\left(a_{1}, a_{2}\right) . \mathfrak{L}(c ; c)$ is called the complete unravelling of $\mathfrak{L}$.

To complete the proof we will show that for $\mathfrak{L}$ as above there is an isotopy of the plane mapping $\mathfrak{L}$ onto $\theta(\mathfrak{L})$. It will suffice to illustrate this in the case where $\mathfrak{L}=\mathfrak{L}(r, s ; t)$ and $r>s$. Let $\mu_{n}$ and $\bar{\mu}_{n}$ denote the semicircles or line segments of $\overline{\mathfrak{L} \cap U}$ and $\overline{\boldsymbol{\Theta}(\mathfrak{L}) \cap U}$ respectively with smallest end point $n$. Similarly define $l_{n}$ and $\bar{l}_{n}$ in $\overline{\mathfrak{L} \cap L}$ and $\overline{\Theta(\mathfrak{L}) \cap L}$ respectively. Consider the curves $\gamma_{n}=l_{n} \cup \mu_{r+n} \cup l_{s-1-n}$ defined for $0 \leq n \leq\|(s-1) / 2\|$. If $s>1$ then $\gamma_{0} \cup \bar{l}_{0}$ bounds a topological disk $D_{0}$ in C. Standard techniques produce an isotopy $f_{0}$ of the plane that differs from the identity only on a neighborhood $N_{0}$ of $D_{0}$ in $\mathbf{C}$ where $f_{0}$ also fixes $\theta(\mathfrak{L}) \backslash \bar{l}_{0}$ and $f_{0}$ maps $\gamma_{0}$ onto $\bar{l}_{0}$. Successively define isotopies $f_{i}$ of the plane taking $\bar{\gamma}_{i}=f_{i-1} \circ \cdots \circ f_{0}\left(\gamma_{i}\right)$ onto $\bar{l}_{i}$ with $f_{i}$ the identity in a region containing $\theta(\mathfrak{L}) \backslash \bar{l}_{i}$. Set $w=\|(s-1) / 2\|$. When $s$ is odd $\gamma_{w}$ will be the union of a semicircle and a ray, and it is clear that there is an isotopy taking $\bar{\gamma}_{s-1}$ onto $\bar{l}_{s-1}$ leaving $\theta(\mathfrak{L}) \backslash \bar{l}_{w}$ fixed. The composition $f_{t} \circ \cdots \circ f_{0}$ maps $\mathfrak{L}$ onto $\theta(\mathfrak{L})$. (In Figure 2.2a the labyrinth $\mathfrak{L}$ on the left is mapped onto $\Theta(\mathfrak{L})$ on the right.)
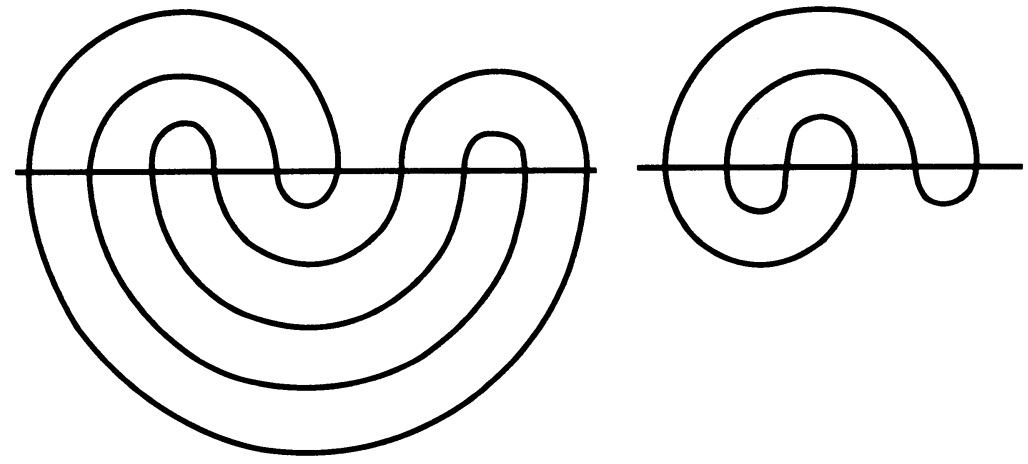

Figure 2.2a. $\theta(\mathfrak{L}(6,4 ; 10))=\mathfrak{L}(6 ; 4,2)$ 
2.4 Lemma. Let $c=\operatorname{gcd}\left(a_{1}+1, a_{2}+1\right)-1$. There is an isotopy of the plane that maps the labyrinth $\mathfrak{L}\left(a_{1}, 1, a_{2} ; b\right)$ onto the labyrinth $\mathfrak{L}(c, 1 ; c ; 1)$ when $c \neq 0$ and onto the labyrinth $\mathfrak{L}(1 ; 1)$ when $c=0$.

As earlier we have two immediate corollaries.

2.5 Corollary. $K\left[\mathfrak{L}\left(a_{1}, 1, a_{2} ; b\right)\right]=\left\|\frac{1}{2}(g+2)\right\|$ where $g=\operatorname{gcd}\left(a_{1}+1, a_{2}+1\right)$.

2.6 Corollary. $\operatorname{gcd}\left(a_{1}+1, a_{2}+1\right)=2 K(\mathfrak{L})-A(\mathfrak{L})$ where $\mathfrak{L}=\mathfrak{L}\left(a_{1}, 1, a_{2} ; b\right)$.

Proof of Lemma 2.4. In order to combine our analysis when $c=0$ and $c>0$ we make the convention that $\mathfrak{L}\left(0,1, a_{2} ; b\right), \mathfrak{L}\left(a_{1}, 1,0 ; b\right), \mathfrak{L}\left(b ; 0,1, a_{2}\right)$ and $\mathfrak{L}\left(b ; a_{1}, 1,0\right)$ stand respectively for the labyrinths $\mathfrak{L}\left(1, a_{2} ; b\right)$, $\mathfrak{L}\left(a_{1}, 1 ; b\right), \mathfrak{L}\left(b ; 1, a_{2}\right)$ and $\mathfrak{L}\left(b ; a_{1}, 1\right)$. We also set $\mathfrak{L}(0,1 ; 0,1)$ to be $\mathfrak{L}(1 ; 1)$.

For a vector $\mathbf{a}=\left(a_{1}, 1, a_{2}\right) \in \mathbf{N}^{3}$ let

$$
\psi(\mathbf{a})= \begin{cases}\left(a_{2}, 1, a_{1}-a_{2}-1\right), & a_{1}>a_{2}, \\ \left(a_{2}-a_{1}-1,1, a_{1}\right), & a_{1}<a_{2}, \\ \left(a_{1}, 1\right), & a_{1}=a_{2} .\end{cases}
$$

We then define an unravelling of the labyrinth $\mathfrak{L}=\mathfrak{L}\left(a_{1}, 1, a_{2} ; b\right)$ by $\theta(\mathfrak{L})=$ $\mathfrak{L}\left(\max \left\{a_{1}, a_{2}\right\} ; \psi(\mathbf{a})\right)$, where $a=\left(a_{1}, 1, a_{2}\right)$. Similarly when $\mathfrak{L}=\mathfrak{L}(b ; \mathbf{a})$ define $\theta(\mathfrak{L})=\mathfrak{L}\left(\psi(\mathbf{a}) ; \max \left\{a_{1}, a_{2}\right\}\right)$. As in the proof of Lemma 2.1 set $\mathfrak{L}_{0}=$ $\mathfrak{L}\left(a_{1}, 1, a_{2} ; b\right)$ and let $\left\{\mathfrak{L}_{i}\right\}$ be the finite sequence of labyrinth with $\mathfrak{L}_{i+1}=$ $\theta\left(\mathfrak{L}_{i}\right)$. The sequence terminates when we reach a labyrinth $\mathfrak{L}_{n}=\mathfrak{L}(c, 1 ; c, 1)$ for some $c \in \mathbf{N}$. Following the reasoning of Lemma 2.1, there is an isotopy of the plane mapping $\mathfrak{L}$ onto $\theta(\mathfrak{L})$. See Figure $2.2(\mathrm{~b})$. We need only show that $c+1=\operatorname{gcd}\left(a_{1}+1, a_{2}+1\right)$.

Define a transformation $\rho\left(a_{1}, 1, a_{2}\right)=\left(a_{1}+1, a_{2}+1\right)$ and $\rho(a, 1)=a+1$. $\rho$ is invertible and we have $\psi=\rho^{-1} \circ \phi \circ \rho$ where $\phi$ is the transformation defined in the proof of Lemma 2.1. As in the earlier argument there is a positive integer $k$ for which $\psi^{k}\left(a_{1}, 1, a_{2}\right)=(c, 1)$ for some integer $c$. Then $\psi^{k-1}\left(a_{1}, 1, a_{2}\right)=(c, 1, c)$ and by the above $\rho^{-1} \circ \phi^{k-1}\left(a_{1}+1, a_{2}+1\right)=$ $\rho^{-1}(c+1, c+1)$. This gives $\phi^{k-1}\left(a_{1}+1, a_{2}+1\right)=(c+1, c+1)$, and by the proof of Lemma 2.1 we conclude that $c+1=\operatorname{gcd}\left(a_{1}+1, a_{2}+1\right)$.

2.2. The next lemma is of use in reducing the connectivity problem for a labyrinth of the form $\mathfrak{L}\left(a_{1}, a_{2} ; b_{1}, b_{2}\right)$ to one already considered.

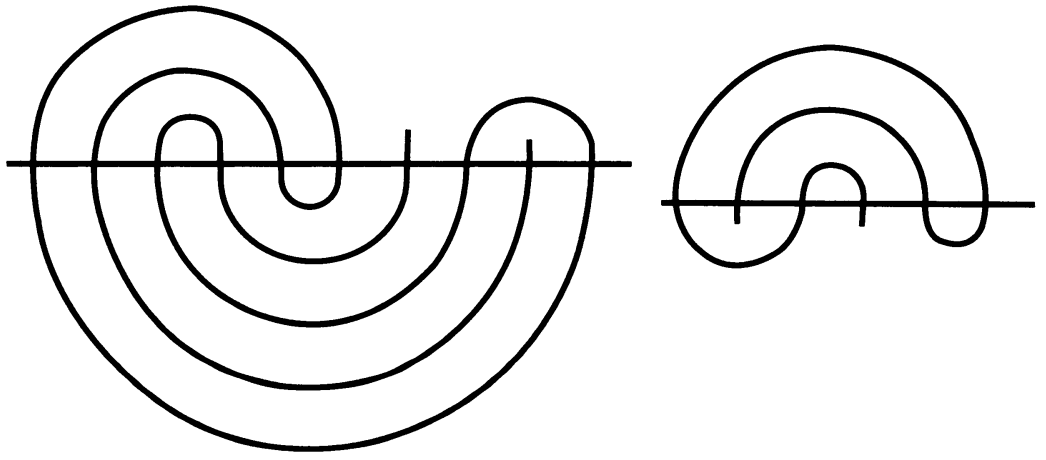

FIGURE 2.2b. $\theta(\mathfrak{L}(6,1,3 ; 10))=\mathfrak{L}(6 ; 3,1,2)$ 
2.7 Lemma. Suppose $a_{1}$ and $b_{1}$ are positive integers with $a_{1} \geq b_{1}$. There is an isotopy $f$ of the Riemann sphere $\widehat{\mathbf{C}}$ that maps the labyrinth $\mathfrak{L}\left(a_{1}, a_{2} ; b_{1}, b_{2}\right)$ onto the labyrinth

$$
\begin{cases}\mathfrak{L}\left(a_{1}-b_{1}, a_{2}+b_{1} ; b_{1}+b_{2}\right) & \text { if } b_{1} \text { is even, } \\ \mathfrak{L}\left(a_{1}-b_{1}-1,1, a_{2}+b_{1}-1 ; b_{1}+b_{2}-1\right) & \text { if } b_{1} \text { is odd. }\end{cases}
$$

If $b_{1}$ is even then the isotopy $f$ may be chosen to preserve the upper and lower half planes. If $b_{1}$ is odd then $f$ may be chosen to preserve $U$ and $L$ except on a small neighborhood of the arcs in $\mathfrak{L}$ attached to the endpoint $\left(b_{1}-1\right) / 2$.

We shall refer to the method for modifying a labyrinth described in Lemma 2.7 as a mound transfer.

Proof. Suppose first that $b_{1}$ is even. Let $u(t)$ (and respectively, $l(t)$ ) be, for $t \in[0,1]$, the semicircle or ray in the upper (and respectively, lower) half plane orthogonal to the real axis with one endpoint at $a_{1}-1$ (and $b_{1}-1$, respectively) and the other endpoint at

$$
x(t)= \begin{cases}2+\frac{1}{t-\frac{1}{2}} & \text { when } 0 \leq t<\frac{1}{2}, \\ \infty & \text { when } t=\frac{1}{2}, \\ \frac{a_{1}+a_{2}}{2 t-1}+1 & \text { when } \frac{1}{2}<t \leq 1 .\end{cases}
$$

Standard techniques in topology give an isotopy $h$ of $\widehat{\mathbf{C}}$ so that

$$
h_{t}(u(0) \cup l(0))=u(t) \cup l(t),
$$

$h$ preserves $U$ and $L$, and $h$ fixes all other points in the labyrinth. The composition of $h$ with a translation to the left by one unit gives an isotopy of the original labyrinth onto $\mathfrak{L}\left(a_{1}-2, a_{2}+2 ; b_{1}-2, b_{2}+2\right)$. Applying the same operation successively $b_{1} / 2$ times results in the labyrinth $\mathfrak{L}\left(a_{1}-b_{1}, a_{2}-\right.$ $\left.b_{1} ; b_{1}+b_{2}\right)$ as stated. The resulting isotopy preserves $U$ and $L$.

When $b_{1}$ is odd perform the operation described above $\left(b_{1}-1\right) / 2$ times. This gives an isotopy of $\mathfrak{L}\left(a_{1}, a_{2} ; b_{1}, b_{2}\right)$ onto $\mathfrak{L}\left(a_{1}-b_{1}+1, a_{2}+b_{1}-1, b_{1}+\right.$ $\left.b_{2}-1\right)$ which preserves $U$ and $L$. The single arc with intersection point 0 in $\mathbf{R}$ may be retracted to an arc in between the two upper mounds resulting in the labyrinth $\mathfrak{L}\left(a_{1}-b_{1}-1,1, a_{2}+b_{1}-1 ; b_{1}+b_{2}-1\right)$. This can be done with an isotopy that preserves $U$ and $L$ except in a neighborhood of the retracted arc.

2.3. We now develop a symbolic scheme for distinguishing the various components in the complement of a labyrinth $\mathfrak{L}$.

Let $\mathfrak{L}$ be one of the labyrinths $\mathfrak{L}\left(a_{1}, a_{2} ; b\right), \mathfrak{L}\left(b ; a_{1}, a_{2}\right), \mathfrak{L}\left(a_{1}, 1, a_{2} ; b\right)$ or $\mathfrak{L}\left(b ; a_{1}, 1, a_{2}\right)$. The fist two of the four classes of labyrinths are said to be of type one, whereas the latter are of type two. Let $\mathfrak{L}^{*}$ be the complete unraveling of $\mathfrak{L}$, and let $h: \mathbf{C} \rightarrow \mathbf{C}$ be the homeomorphism taking $\mathfrak{L}$ to $\mathfrak{L}^{*}$.

Label the unbounded component of $\mathbf{C} \backslash \mathfrak{L}^{*}$ zero. The component adjacent to it is labeled one. Continue labeling components so that the $i$ th component is contiguous to the $(i-1)$ th and the $(i+1)$ th components for $0 \leq i \leq K-A$ where $K$ and $A$ are defined in $\S 2.1$. The labeling of the components of $\mathbf{C} \backslash \mathfrak{L}^{*}$ induces a labeling of the components of $\mathbf{C} \backslash \mathfrak{L}$ by way of the map $h$. 
The integers where $\mathfrak{L}$ meets $\mathbf{R}$ divide the real line into intervals which are naturally ordered beginning at the left with zero and continuing consecutively up to the $b$ th interval. Define a function $\Sigma_{\mathfrak{L}}: \mathbf{Z}(b) \rightarrow \mathbf{Z}(K-A)$ where $\Sigma_{\mathfrak{L}}(x)$ is the label of the component of $\mathbf{C} \backslash \mathfrak{L}$ containing the $x$ th interval in $\mathbf{R} \backslash \mathfrak{L}$, and where $\mathbf{Z}(j)=\{x \in \mathbf{Z} \mid 0 \leq x \leq j\}$.

Let $\alpha: \mathbf{Z}(K-A) \rightarrow \mathbf{Z}(K-A)$ be the identity function and set $\bar{\alpha}(x)=$ $\alpha(K-A-x)=K-A-x$. Given functions $\Sigma_{1}: \mathbf{Z}\left(n_{1}\right) \rightarrow \mathbf{Z}(K-A)$ and $\Sigma_{2}: \mathbf{Z}\left(n_{2}\right) \rightarrow \mathbf{Z}(K-A)$ we define two products: $\Sigma_{1} \circ \Sigma_{2}: \mathbf{Z}\left(n_{1}+n_{2}+1\right) \rightarrow \mathbf{Z}(K-A)$ by

$$
\Sigma_{1} \circ \Sigma_{2}(x)= \begin{cases}\Sigma_{1}(x) & \text { for } 0 \leq x \leq n_{1}, \\ \Sigma_{2}\left(x-n_{1}-1\right) & \text { for } n_{1}<x \leq n_{1}+n_{2}+1\end{cases}
$$

and $\Sigma_{1} \times \Sigma_{2}: \mathbf{Z}\left(n_{1}+n_{2}\right) \rightarrow \mathbf{Z}(K-A)$ by

$$
\Sigma_{1} \times \Sigma_{2}(x)= \begin{cases}\Sigma_{1}(x) & \text { for } 0 \leq x<n_{1}, \\ \Sigma_{2}\left(x-n_{1}\right) & \text { for } n_{1} \leq x \leq n_{1}+n_{2} .\end{cases}
$$

Let $\Sigma^{\circ n}$ and $\Sigma^{\times n}$ denote respectively the $n$-fold products $\Sigma \circ \ldots \circ \Sigma$ and $\Sigma \times$ $\cdots \times \Sigma$.

We define a function $\sigma$ by concatenating $\alpha$ and $\bar{\alpha}$ in various different fashions depending on the type of $\mathfrak{L}$ and the value of $A$. We set $\sigma=\alpha \times \bar{\alpha}$ if either $\mathfrak{L}$ is of type one with $A=0$ or if $\mathfrak{L}$ is of type two with $A=1$. Otherwise set $\sigma=\alpha \circ \bar{\alpha}$.

2.8 Lemma. Given $\mathfrak{L}$ as above with $\mathbf{C} \backslash \mathfrak{L}$ not connected there is a positive integer $l$ so that $\Sigma_{\mathfrak{L}}=\sigma^{\times l}$ if $\mathfrak{L}$ is of type one and $\Sigma_{\mathfrak{L}}=\sigma^{\circ l}$ if $\mathfrak{L}$ is of type two. When $\mathbf{C} \backslash \mathfrak{L}$ is connected, $\Sigma_{\mathfrak{L}} \equiv 0$.

Proof. Consider the sequence of unravellings $\mathfrak{L}=\mathfrak{L}_{0}, \mathfrak{L}_{1}, \ldots, \mathfrak{L}_{q}=\mathfrak{L}^{\prime}$ described in Lemmas 2.1 and 2.4 where $\mathfrak{L}^{\prime}=\mathfrak{L}^{*}$ if $\mathfrak{L}$ is of type one and where $\mathfrak{L}^{\prime}$ is of one of the forms $\mathfrak{L}(c, 1, c ; 2 c+1)$ or $\mathfrak{L}(2 c+1 ; c, 1, c)$ if $\mathfrak{L}$ is of type two. In other words, for a type two labyrinth, $\theta\left(\mathfrak{L}^{\prime}\right)$ is the complete unraveling of $\mathfrak{L}$.

We will argue by induction on $q$. When $q=0, \mathfrak{L}=\mathfrak{L}^{\prime}$ and it is easy to verify that $\Sigma_{\mathfrak{L}^{\prime}}$ is of the form described in the lemma. Now suppose that the lemma is true for labyrinths for which $q \leq n$ and let $\mathfrak{L}$ be a labyrinth with $q=n+1$.

Consider the two mounds of $\mathfrak{L}$ labeled $a_{1}$ and $a_{2}$. The larger mound will become the single isolated mound of the initial unravelling $\mathfrak{L}_{1}$ and so, by the inductive hypothesis, it is described by a function $\Sigma_{1}$, as in the statement of the lemma. The smaller mound will also eventually become either the single isolated mound of $\mathfrak{L}_{i}$ for some $i$ with $0 \leq i \leq n$ or one of the two mounds of $\mathfrak{L}_{q}$. In both cases it is clearly described by a function $\Sigma_{2}$, of the desired form. $\Sigma_{\mathfrak{L}}$ will then be one of the products $\Sigma_{1} \times \Sigma_{2}$ or $\Sigma_{2} \times \Sigma_{1}$ if $\mathfrak{L}$ is of type one and $\Sigma_{1} \circ \Sigma_{2}$ or $\Sigma_{2} \circ \Sigma_{1}$ if $\mathfrak{L}$ is of type two. The result follows.

2.9 Lemma. Let $\mathfrak{L}$ be as above with connectivity $K$ and $A$ components with boundary. If $\mathfrak{L}$ has type one then

$$
\Sigma_{\mathfrak{L}}(x)= \begin{cases}x_{0} & \text { if } 0 \leq x_{0}<K, \\ 2 K-A-x_{0} & \text { if } k \leq x_{0}<2 K-A,\end{cases}
$$

and if $\mathfrak{L}$ has type two then 


$$
\Sigma_{\mathfrak{L}}(x)= \begin{cases}x_{0} & \text { if } 0 \leq x_{0}<K+1-A, \\ 2 K-1-A-x_{0} & \text { if } K+1-A \leq x_{0}<2 K-A,\end{cases}
$$

where $x_{0} \equiv x \bmod (2 K-A)$ and $0 \leq x_{0}<2 K-A$.

Proof. The case where $\mathbf{C} \backslash \mathfrak{L}$ is connected corresponds to either $K=1$ and $A=1$ or $K=2$ and $A=2$. The formulas are easily verified. It follows from the previous lemma that in all other cases the function $\Sigma_{\mathfrak{L}}$ is periodic of period $2 K-A$. It therefore suffices to consider the restriction of $\Sigma_{\mathfrak{L}}$ to $\mathbf{Z}(2 K-A-1)$. When $\mathfrak{L}$ has type one and $A=0, \Sigma_{\mathfrak{L}}=\alpha \times \bar{\alpha}$ on $\mathbf{Z}(2 K-1)$. For $0 \leq x \leq K$ we get $\Sigma_{\mathfrak{L}}(x)=\alpha(x)=x$ and for $K \leq x<2 K, \Sigma_{\mathfrak{L}}(x)=$ $\bar{\alpha}(x-K)=\alpha(2 K-x)=2 k-x$ as required. When $\mathfrak{L}$ has type two and $A=2$, $\Sigma_{\mathfrak{L}}=\alpha \circ \bar{\alpha}$ on $\mathbf{Z}(2 K-3)$. Then for $0 \leq x \leq K-2, \Sigma_{\mathfrak{L}}(x)=\alpha(x)=x$ and for $K-2<x \leq 2 K-3, \Sigma_{\mathfrak{L}}(x)=\bar{\alpha}(x-(K-2)-1)=2 K-3-x$ as required. We leave the remaining cases for the reader to verify.

2.10 Remark. Lemma 5.2 may be condensed to give

$$
\Sigma_{\mathfrak{L}}(x)= \begin{cases}x_{0} & \text { if } 0 \leq x_{0}<\left\|\frac{2 K-A+1}{2}\right\|, \\ 2 K-A-D-x & \text { if }\left\|\frac{2 K-A+1}{2}\right\| \leq x \leq 2 K-A,\end{cases}
$$

and $x_{0}=x \bmod 2 K-A$ where $D$ takes the value zero when $\mathfrak{L}$ is type one and the value one when $\mathfrak{L}$ is type two.

We will begin this section by translating the train track problem on $S$ into the language of labyrinths. There is no loss of generality in assuming that the train track $\tau_{0}$ on $S$ lies in the plane and is symmetric about the origin. (There is actually a symmetry of $S$ defined on $D$ and $D^{\prime}$ by $z \mapsto-z$ that maps $\tau_{0}$ onto itself.) Label the branch points on $S b_{1}, b_{2}, \ldots, b_{6}$ where the first four are vertices of $\tau_{0}$ and $b_{i}$ is in the $i$ th quadrant (see Figure 1.3d). The branch point in the bounded region in the complement of $\tau_{0}$ will be labelled $b_{5}$ and the remaining branch point in the unbounded region $b_{6}$. We may also assume that $b_{5}$ lies in the upper half plane and $b_{6}$ in the lower half plane.

When $n=0$ the train track $\tau$ on $N$ reduces to a union of sine curves of weight $k$ around the midsection of $N$. Henceforth, we assume that $n \neq 0$.

A representative of the multiarc $M_{0}(n, t, v, k)$ can clearly be deformed into a concentric labyrinth $\overline{\mathfrak{L}}=\mathfrak{L}(2 v-t+2 k, n-v ; v, n-t+2 k)$. By standard techniques in topology there is a homeomorphism $f: \widehat{\mathbf{C}} \rightarrow \widehat{\mathbf{C}}$ mapping $M_{0}$ onto $\overline{\mathfrak{L}}$. We may further suppose that the representative multicurve $M_{0}$ has been chosen to have as few intersections with $\mathbf{R}$ as possible and that $f$ preserves the upper and lower half planes. These last conditions allow us to keep track of the branch points.

To further simplify things we shall subject $\overline{\mathfrak{L}}$ to a mound transfer. The result is a labyrinth

$$
\mathfrak{L}=\left\{\begin{array}{l}
\mathfrak{L}(v-t+2 k, n ; n+v-t+2) \quad \text { when } v \text { is even } \\
\mathfrak{L}(v-t+2 k-1,1, n-1 ; n-v-t+2 k-1) \text { when } v \text { is odd }
\end{array}\right.
$$

Combining Lemma 2.7 with the above gives a homeomorphism $g: \widehat{\mathbf{C}} \rightarrow \widehat{\mathbf{C}}$ mapping $M_{0}$ onto $\mathfrak{L}$. When $v$ is even, $g$ can be chosen to preserve the upper and lower half planes. When $v$ is odd, $g$ can be chosen to preserve $L$ and $U$, except in a small neighborhood of an arc terminating at $b_{3}$ which contains 
no other branch points. From now on we shall identify $M_{0}$ with the labyrinth $\mathfrak{L}$.

It is important that we get a precise fix on the position of the branch points $b_{5}$ and $b_{6}$ with respect to $\mathfrak{L}=M_{0}$. The components of $U \backslash \mathfrak{L}$ and $L \backslash \mathfrak{L}$ that contains branch points may be designated by the numbering (as introduced in $\S 2.3)$ of one of the intervals on its boundary. Let $V_{5}$ be the region in $U \backslash \mathfrak{L}$ containing $b_{5}$ and let $V_{6}$ be the region in $L \backslash \mathfrak{L}$ containing $b_{5}$. Then one of the intervals on the boundary of $V_{5}$ is numbered $v / 2+k$ when $v$ is even and $(v-1) / 2+k$ when $v$ is odd. Similarly one of the intervals on the boundary of $V_{6}$ is numbered $v / 2$ when $v$ is even and $(v-1) / 2$ when $v$ is odd. In order to see this one should first note the positions of the points $b_{5}$ and $b_{6}$ with respect to the labyrinth $\overline{\mathfrak{L}}$ and then watch how they are affected by the mound transfer.

3.1. Let $T$ be a hyperelliptic Riemann surface of genus $g$ with hyperelliptic involution $\phi$. The quotient of $T$ by $\phi$ is a sphere $S$ and the projection $\pi: T \rightarrow S$ is branched over $2 g+2$ points. Let $B$ denote the set of branch points on $S$.

Let $\alpha$ be a simple closed curve on the punctured sphere $S \backslash B$, and let $\tilde{\alpha}$ be the preimage of $\alpha$ on $T$. Since $\pi$ is a 2-sheeted cover $\tilde{\alpha}$ will consist of either two disjoint simple closed curves each of which is mapped homeomorphically onto $\alpha$ by $\pi$, or a single simple closed curve that is a double cover of $\alpha$. The following is an easy exercise in covering theory.

3.1 Proposition. Let $\alpha$ be a simple closed curve on $S \backslash B$ that bounds a region $D$ containing $b$ punctures. Then the preimage $\tilde{\alpha}$ of $\alpha$ on $T$ is connected if and only if $b$ is odd.

3.2. In this subsection we will complete the proof of Theorem 1.1 .

3.2 Proposition. The number of connected components of $M(n, t, v, k)$ is $\operatorname{gcd}(v-t+2 k, n)-R$ where $R$ denotes the number of components of $M_{0}(n, t, v, k)$ that enclose a region on $S$ containing exactly three branch points.

Proof. We identify $M_{0}$ with the labyrinth $\mathfrak{L}$ as in $\S 3.0$. Applying Corollaries 2.3 and $2.6 \operatorname{gives} \operatorname{gcd}(v-t+2 k, n)=2 K-A$ where we recall that $K$ is the number of connected components of $M_{0}$ and $A$ is the number of components with boundary (either zero, one, or two). Note that with the current set up these arcs must join branch points on $S$.

It is evident that the connectivity of $M$ is twice the connectivity of $M_{0}$ minus the number of component of $M_{0}$ with connected preimages on $N$. The $A$ arcs of $M_{0}$ joining branch points all have connected preimages on $N$. By Proposition 3.1 the only other components with connected preimages are those that bound a region on $S$ containing an odd number of the branch points, that is either one branch point or three branch points.

The proof will be completed by showing that no component of $M_{0}$ bounds a region on $S$ containing exactly one branch point. Without loss of generality suppose that $M_{0}$ has a component that is a closed 1-manifold. The above assertion will follow if we show that the inner and outermost regions in the complement of $M_{0}$ each contains at least two branch points. In particular, since the branch point $b_{3}$ lies in the unbounded region in the complement of the labyrinth, it will suffice to show that of the branch points $b_{1}, b_{2}$ and $b_{4}$ 
two lie in the innermost and one in the outermost region in the complement of $M_{0}$.

Let $M_{0}=\mathfrak{L}_{0}, \mathfrak{L}_{1}, \ldots, \mathfrak{\sim}_{q}=\mathfrak{L}^{*}$ be the sequence of unravellings for $M_{0}$ where $\mathfrak{L}^{*}$ is the complete unravelling. The innermost semicircle of a mound bounds a half-disc in either $U$ or $L$, which we shall refer to as the half-disc of the mound. It follows from Corollaries 2.3 and 2.6 that for each $i<q, \mathfrak{L}_{i}$ has three semicircular mounds, and thus there are three half-discs. The unravelling of $\mathfrak{L}_{i-1}$ to $\mathfrak{L}_{i}$ is realized by a homeomorphism of $\mathbf{C}$ that maps the three halfdiscs of $\mathfrak{L}_{i-1}$ onto the half-discs of $\mathfrak{L}_{i}$. Since each half-disc of $\mathfrak{L}_{0}$ contains one of the branch points $b_{1}, b_{2}, b_{4}$ it follows that the half-discs of $\mathfrak{L}_{i}$ with $i<q$ all contain branch points.

The penultimate labyrinth $\mathfrak{L}_{q-1}$ is of the form $\mathfrak{L}(a, a ; b), \mathfrak{L}(b ; a, a)$, $\mathfrak{L}(a, 1, a ; b)$, or $\mathfrak{L}(b ; a, 1, a)$. Thus two of the half-discs of $\mathfrak{L}_{q-1}$ lie in the region mapped to the interior of the concentric circles of $\mathfrak{L}_{q}$. The half-disc associated with the middle mound of $\mathfrak{L}_{q-1}$ lies in the unbounded region in the complement of $\mathfrak{L}_{q-1}$ and will therefore map onto the unbounded region in the complement of $\mathfrak{L}_{q}$. The result follows.

3.3 Remark. The last part of the above argument shows that two of the branch points $b_{1}, b_{2}, b_{3}, b_{4}$ lie in the complementary region of $M_{0}$ labeled 0 and the other two lie in the region labeled $K-A$.

We shall now derive a formula for $R$ thereby completing the proof of Theorem 1.1. Let $\mathfrak{L}$ be one of the labyrinths of $\S 2.3$ and let $\mathfrak{L}^{*}$ be the complete unravelling of $\mathfrak{L}$. Label the complementary components of $\mathfrak{L}$ and $\mathfrak{L}^{*}$ as in $\S 2.3$. The labyrinths are to be viewed (up to isotopy) as lying in the branched sphere $S$ so that two of the branch points lie in the region labeled zero and two lie in the region labeled $K-A$. The two remaining branch points are assumed to belong respectively to regions labeled $r_{1}$ and $r_{2}$ where $r_{1} \leq r_{2}$.

The circles (if any) in $\mathfrak{L}^{*}$ that bound a region containing three of the branch points are those sandwiched in between the regions $r_{1}$ and $r_{2}$. Their number is $r_{2}-r_{1}$. Since $\mathfrak{L}$ and $\mathfrak{L}^{*}$ are identified by a homeomorphism of $\widehat{\mathbf{C}}$ the number of closed curves in $\mathfrak{L}$ that bound a region containing three branch points is also $r_{2}-r_{1}$.

When $\mathfrak{L}$ is the labyrinth $M_{0}(n, t, v, k)$ we have already seen in Remark 3.3 , that two of the branch points $b_{1}, b_{2}, b_{3}, b_{4}$ are in the 0th component and the other two are in the $(K-A)$ th component. The above observation therefore applies with $r_{1}$ and $r_{2}$ denoting respectively the regions containing $b_{5}$ and $b_{6}$, giving $R=\left|r_{1}-r_{2}\right|$. Recall that, the label of a region in the complement of the labyrinth containing a branch point $b_{i}$ will be the same for both $\mathfrak{L}$ of $\mathfrak{L}^{*}$. In $\S 3.0$ we determined intervals of $\mathbf{R} \backslash \mathfrak{L}$ belonging to the same complementary regions as $b_{5}$ and $b_{6}$ respectively. Evaluating the function $\Sigma_{\mathfrak{L}}$ at these values will then give the labels for the regions containing $b_{5}$ and $b_{6}$. We have $R=\left|\Sigma_{\mathfrak{L}}\left(\frac{v}{2}+k\right)-\Sigma_{\mathfrak{L}}\left(\frac{v}{2}\right)\right|$ when $v$ is even and $R=\left|\Sigma_{\mathfrak{L}}\left(\frac{v-1}{2}+k\right)-\Sigma_{\mathfrak{L}}\left(\frac{v-1}{2}\right)\right|$ when $v$ is odd. Writing $\Sigma_{\mathfrak{L}}$ as in Lemma 2.9 and Remark 2.10 gives the main theorem.

We now restrict our attention to multiple curves $M(n, t, v, k)$ on $N$ which are connected and we use the results of the previous section to prove Theorems 
1.2 and 1.3. Recall that $G=\operatorname{gcd}(v-t+2 k, n)$ is twice the connectivity of $M_{0}$ minus the number of arcs connecting branch points and $R$ is the number of closed curves of $M_{0}$ that enclose exactly three branch points of $S$.

4.1 Proof of Theorem 1.2. Suppose $M$ is connected. Then by Proposition 3.1 and the Remark 3.3, $M$ projects either to a closed curve enclosing exactly three branch points of $S$ or to an arc connecting two branch points of $S$. In the two cases we have respectively $G=2$ and $R=1$ or $G=1$ and $R=0$. When $G=2, n$ is even and since $M_{0}$ has no arcs connecting branch points, all of the parameters $u, s, v$, and $t$, in particular $v$ and $t$, are even. In this case the function $R$ reduces to $R=k \bmod 2$. Therefore $k$ is odd.

Conversely, suppose $G=2, t$ and $v$ are even, and $k$ is odd. Since $n, t$ and $v$ are even $M_{0}$ is a closed curve. Since $k$ is odd $R=1$ and $M_{0}$ encloses three branch points. Therefore by Proposition 3.1 $M$ is connected. If $G=1$ then $M_{0}$ is an arc connecting two branch points of $S$ and $M_{0}$ lifts to a connected curve on $M$.

The assertion that $M$ is nondividing if and only if $G=1$ follows from the easy observation that the preimage of an arc joining branch points is a nondividing curve.

4.2 Proof of Theorem 1.3. Consider the multiple curves $M(k)=M(n, t, v, k)$ with $n, t$ and $v$ fixed. Note that both $G$ and $R$ are $2 n$ periodic in $k$. Let $w$ be a fixed integer where $w \geq t-v$. Since the connectivity of $M$ is given by $G-R$, the connectivity of the curves $M(k)$ for integers $k \geq w$ is $2 n$ periodic. Moreover, the curve $M(k+2 n m)$ is obtained from $M(k)$ by performing $m$ Dehn twists about the curve on $N$ formed by the branches of $\tau$ labelled $k$ and $v-t+k$.

We are interested in computing the number of $M(k)$ that are connected in one $2 n$ period. Choose $q$ so that $q n \geq t-v$. We shall compute the number of connected curves $M(k)$ for $k$ in the set $Z=\{k: q n \leq k \leq q n+2 n-1\}$. There are six cases which exhaust the possibilities.

Case I. Suppose $n$ is odd. Then the curve $M(k)$ is connected exactly when $\operatorname{gcd}(v-t+2 k, n)=1$. For values of $k$ from $q n$ to $q n+(n-1) / 2$ the values of $v-t+2 k$ are given by $v-t+2 q n+i, i=0,2,4, \ldots, n-1$. For values of $k$ from $q n+(n-1) / 2+1$ to $q n+n-1$ the values of $v-t+2 k$ are given by $v-t+2 q n+i, i=n+1, n+3, \ldots, 2 n-2$. Since $\operatorname{gcd}(m+n, n)=\operatorname{gcd}(m, n)$ for all $m$ and $n$, we may add multiples of $n$ to the values $v-t+2 q n+i$ above without changing their greatest common divisors with $n$. In the former case we add $-2 q n$ to each value $v-t+2 q n+i$ and in the latter case we add $-2 q n-n$ to each value of $v-t+2 q n+i$. Combining we obtain the values $v-t+j, j=0, \ldots, n-1$. There are $\phi(n)$ values of $v-t+j$ for $0 \leq j<n$ such that $\operatorname{gcd}(v-t+j, n)=1$. Therefore there are $\phi(n)$ values of $k$ for $k \in\{q n, \ldots, q n+n-1\}$ for which $\operatorname{gcd}(v-t+2 k, n)=1$. The same argument holds for $k \in\{q n+n, \ldots, q n+2 n-1\}$. Therefore there are $2 \phi(n)$ values of $k \in Z$ for which $M(k)$ is connected.

Case II. Suppose $n, v$, and $t$ are even and $n / 2$ is odd. Then

$$
\operatorname{gcd}(v-t+2 k, n)=2 \operatorname{gcd}((v-t) / 2+k, n / 2) .
$$

Since $\operatorname{gcd}(v-t+2 k, n)>1$, by Theorem $1.2 M(k)$ is connected if and only if $\operatorname{gcd}((v-t) / 2+k, n / 2)=1$ and $k$ is odd. Now, because $\phi$ is multiplicative for 
relatively prime integers, there are $4 \phi(n / 2)=4 \phi(2) \phi(n / 2)=4 \phi(n)$ values of $k \in Z$ for which $\operatorname{gcd}((v-t) / 2+k, n / 2)=1$. Since $n$ is even and $n / 2$ is odd we conclude that in exactly $2 \phi(n)$ of the $4 \phi(n)$ cases where $\operatorname{gcd}((v-t) / 2+$ $k, n / 2)=1,(v-t) / 2+k$ is odd and in the $2 \phi(n)$ remaining cases $(v-t) / 2+k$ is even. Therefore in $2 \phi(n)$ of the $4 \phi(n)$ cases where $\operatorname{gcd}((v-t) / 2+k, n)=1$, $k$ is odd. Thus $M(k)$ is connected for $2 \phi(n)$ values of $k \in Z$.

Case III. Suppose $n, n / 2, t, v$ and $(v-t) / 2$ are even. Then

$$
\operatorname{gcd}(v-t+2 k, n)>1
$$

and by Theorem $1.2 M(k)$ is connected if and only if $\operatorname{gcd}(v-t+2 k, n)=$ 2 and $k$ is odd. Then $\operatorname{gcd}(v-t+2 k, n)=2 \operatorname{gcd}((v-t) / 2+k, n / 2)$ and $\operatorname{gcd}((v-t) / 2+k, n / 2)=1$ for $4 \phi(n / 2)$ values of $k \in Z$. Since $n / 2$ is even, $4 \phi(n / 2)=2 \phi(n)$. Also since $(v-t) / 2$ and $n / 2$ are even,

$$
\operatorname{gcd}((v-t) / 2+k, n / 2)=1
$$

occurs only when $k$ is odd. Therefore there are $2 \phi(n)$ values of $k \in Z$ for which $M(k)$ is connected.

Case IV. Suppose $n$ is even and $v-t$ is odd. Then by Theorem $1.2 M(k)$ is connected if and only if $\operatorname{gcd}(v-t+2 k, n)=1$. Since $v-t+2 k$ is odd for all $k, v-t+2 k$ takes on every odd value $\bmod n$ four times as $k$ goes from $q n$ to $(q+2) n-1$. There are no even integers $m$ for which $\operatorname{gcd}(m, n)=1$ and there are $4 \phi(n)$ values of $k$ in $Z$ for which $\operatorname{gcd}(v-t+2 k, n)=1$, so for $4 \phi(n)$ values of $k$ in $Z, M(k)$ is connected.

Case V. Suppose $n$ is even, and both $t$ and $v$ are odd. Then $v-t+2 k$ is even and $\operatorname{gcd}(v-t+2 k, n)>1$. By Theorem 1.2 there are no multicurves $M(k)$ which are connected.

Case VI. Suppose $n, n / 2, t, v$ are even and $(v-t) / 2$ is odd. Since

$$
\operatorname{gcd}(v-t+2 k, n)>1 \text {, }
$$

$M(k)$ is connected if and only if $\operatorname{gcd}(v-t+2 k, n)=2$ and $k$ odd. As above $\operatorname{gcd}(v-t+2 k, n)=2 \operatorname{gcd}((v-t) / 2+k, n / 2)$ and

$$
\operatorname{gcd}((v-t) / 2+k, n / 2)=1
$$

only if $(v-t) / 2+k$ is odd. Since $(v-t) / 2$ is odd this occurs when $k$ is even. Therefore $M(k)$ is not connected for any $k$.

\section{REFERENCES}

1. A. Haas and P. Susskind, The geometry of the hyperelliptic involution in genus two, Proc. Amer. Math. Soc. 105 (1989), 159-165.

2. R. C. Penner, An introduction to train tracks, Low-dimensional Topology and Kleinian Groups, Warwick and Durham, 1984, (Epstein, ed.), LMS Lecture Notes Ser. 112, Cambridge Univ. Press, Cambridge and New York, 1986, pp. 77-90.

3. J. Harer and R. C. Penner, Combinatories of train tracks, unpublished manuscript.

4. W. Thurston, The geometry and topology of 3-manifolds, Princeton Univ. Notes.

Department of Mathematics, University of Connecticut, Storrs, Connecticut 06268

Department of Mathematics, Connecticut College, New London, Connecticut 06320 